\title{
STRESSES AROUND A CIRCULAR HOLE IN A SHALLOW CONICAL SHELL WITH TORSIONAL LOADING
}

\author{
Ramish Chandrat and B. BaSAVA Raju \\ Naltumal Aeronautical Laboratory, Bangulore-17, India
}

\begin{abstract}
Antyliul solutions are presented for the stresses in u conical shell having a circular hole on its lateral surface. The shell is suluisted to tursional load, The method of analysis involves perturbations in parameters defining curvilure and the cone angle of the shell $(\beta$ and $r$. respectively). The membrane and bending stresses are obtuined retaining terms of the order of $\beta^{i}$ and $\ell^{2}$.
\end{abstract}

\section{INTRODUCTION}

TH: stresses around a circular hole in a conical shell have been determined with the axial tension and internal pressure loading retaining terms of $f t^{2}$ and $\iota^{2}$ order [1]. It has been noliced [ I] that retaining lerms of $s^{2}$ order is not very useful in the sense that conical shell solutions are not very much dilTercnt from cylindrical ones, unless higher order terms in $\beta$ are also retained. It appears from the correlation between these parameters $\theta$ and $f i$ that \&: is of $/ \beta^{2}$ arder provided $r_{6} / h ? 242 \tan \alpha(\mu=0.3)$. In view of the above correlation between the orders of these parameters, it becomes essential to consider the terms of $\beta^{4}$ order at lealst, if one decides to consider the $\varepsilon^{2}$ order terms. This has motivated the present investigation, where solutions are attempted, retaining terms of $\beta^{4}$ and $\varepsilon^{2}$ order for the torsional loading. Formulae, from which the membrane and bending stresses can be computed, are presented and numerical results are given for various values of these parameters.

\section{THE GOVERNING EQUATIONS}

\subsection{The differenticikequation}

Notation is the same as used in [1].\$ The differential equation for a thin conical shell is obtained from [1] as follows:

$$
v^{4} \phi+\frac{8 i f^{2} \partial^{2} \phi}{\partial s^{2}}=0
$$

\section{2,2, Boundurv conditions}

The boundary conditions at $\mathrm{r}$ man 1 are

$$
\begin{array}{ll}
N_{r}^{T}=0, & N_{r 0}^{r}-0 \\
M_{r}=0, & Q_{r}^{*}-0 .
\end{array}
$$

"S Seientist, Structural Sciences Division.

Head, Structural Sciences Division.

\& In [1] Poisson's ratio has been denoted by v, but in the present Paper it is denoted by $\mu$. 


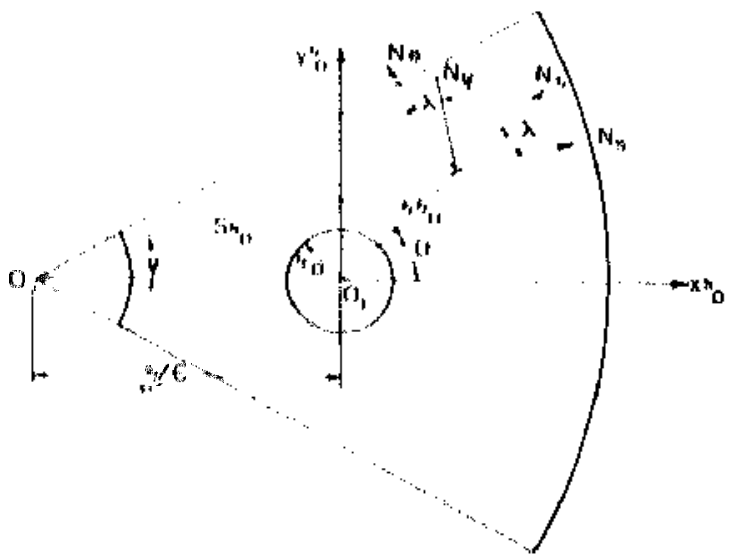

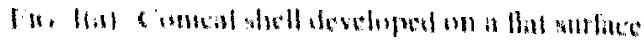
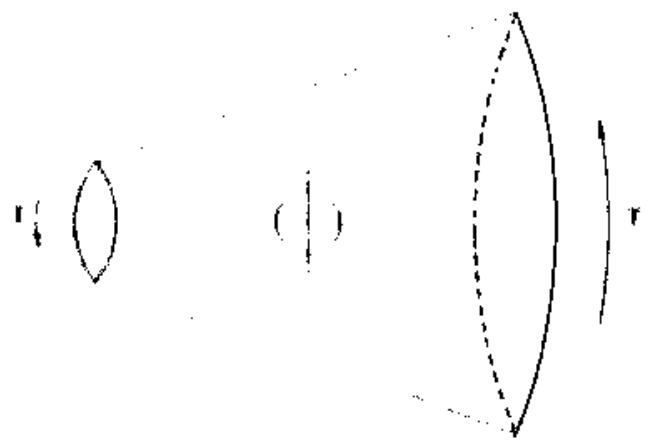

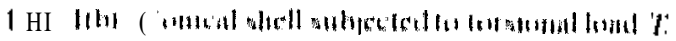

\section{MEIHIOD OF ANALYSIS}

The methad of analyas invelvos perturbations with respect to the parameter $\beta$ i id \& The hroke wisumed to he small enough sin that // * I, It can he shown that (i is the nater of $f^{3}$ if

$$
r_{11} \cdot 2 \cdot 42 \operatorname{ann} x(11 \cdots(1.3)
$$

Nisw the complex stess displatement function and all the stress resultants $\mathrm{C} i$ be

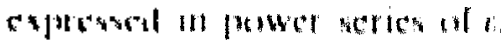

$$
\begin{aligned}
& \phi \cdots \phi_{n}+k_{1} k^{2} \phi_{n} \\
& N_{r} \ldots N_{r 11}+1 N_{r 1}+1^{2} N_{r 2} \text { ats. }
\end{aligned}
$$

Futher, cach txrm th the puwer series coun he expressed in even powers of $B$, and pro< cts

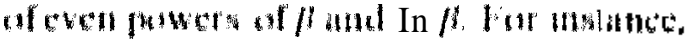

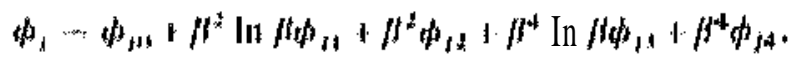


In this paper, we are retaining the terms of the order of $\beta^{4}$. The terms of $\beta^{6} \ln \beta$ order and higher have been neglected. Substituting $j=0,1$ and 2 , in the above expression,

$$
\begin{aligned}
& 00=00<+\beta^{2} \ln \beta \phi_{01}+\beta^{2} \phi_{02}+\beta^{4} \ln \beta \phi_{03}+\beta^{4}{ }_{{ }_{\mathrm{P} 04}} \\
& \phi_{1}=\phi_{10}+\beta^{2} \ln \beta \phi_{11}+\beta^{2} \phi_{12}+0\left(\beta^{4} \ln \beta\right) \\
& \phi_{2}=\phi_{20}+0\left(\beta^{2} \ln \beta\right) .
\end{aligned}
$$

As ;; is of the order of $f t^{2}$, we can neglect the term of the order of $\beta^{4} \ln \beta$ in the expansion of $\phi_{1}$ and terms of the order of $f t^{2} \ln \beta$ in the expansion of $\phi_{2}$.

\section{SOLUTION FOR SMALL VALUES OF $f t$ AND e}

The membrane and bending solution will be obtained by considering only the first three terms of series in the expression (3). For this approximation, equation (1) reduces to three differential equations for $\phi_{0}, \phi_{1}, \phi_{2}$. These equations are the same as equations (1 la), (11 b) and (11c) of [1]. The complementary solution of any of these equations is given below:

$$
\phi_{j}=\sum_{n=1}^{n+1}\left(A_{n 1}+i B_{n 1}\right)\left(\alpha_{n 1}+i / \beta_{n 1}\right)+\sum_{n=1}^{\infty}\left(A_{n 2}+i B_{n 2}\right)\left(\alpha_{n 2}+\beta_{n 2}\right)
$$

where

$$
\begin{aligned}
& \alpha_{n 1}+i \beta_{n 1}=\cosh [(1-i) \beta x] H_{n}^{\mathrm{t}}(\sqrt{ } 2 i f i r) \sin n O \\
& \alpha_{n 2}+i \beta_{n 2}=\sinh [(1-i) \beta x] H_{n}^{1}(\sqrt{ } 2 f t r) \sin n O
\end{aligned}
$$

\subsection{Modifiedboundary conditions}

The boundary conditions as defined in Section 2.2 are reformulated in this section. The membrane boundary conditions at $r=1$ are formulated as follows:-

where $j=0,1,2$.

$$
\begin{array}{r}
N_{r j}+\bar{N}_{r j}=0 \\
N_{r 0 j}+\bar{N}_{r 0 j}=0
\end{array}
$$

Substituting for $N_{r j}$ and $N_{r 0 j}$ from [1] and $\bar{N}_{r j}$ and $N_{r 0 j}$ from Appendix in the above equations, following boundary conditions for zeroth, first and second order approximation in $\varepsilon$ are obtained

Zeroth approximation:

$$
\begin{aligned}
\left(\begin{array}{ll}
1 & 0 \\
r & \frac{1}{r}+\frac{1}{r^{2}} \frac{\partial^{2}}{\partial \theta^{2}}
\end{array}\right) \operatorname{Im} \phi_{0 k}=m r_{0}^{2} \tau_{0} \sin 20, \quad k=0 \\
=0, \quad k-1,2,3,4 \ldots \\
\frac{\partial}{\partial r}\left(\frac{1}{r} \frac{d}{\partial \theta}\right) \operatorname{Im} \phi_{0 k} \quad=-m r_{0}^{2} \tau_{0} \cos 20, \quad k=0
\end{aligned}
$$

First approximation:

$$
\begin{aligned}
& \left(\begin{array}{ll}
\left.\frac{1}{\mathrm{r}} \frac{\partial}{\partial r}+\frac{1}{r^{2}} \frac{\partial^{2}}{\partial \theta^{2}}\right) \operatorname{Im} \phi_{1 k} & =-2 m r_{0}^{2} \tau_{0} \sin 30, \quad k=0 \\
\frac{\partial}{d r}\left(\frac{1}{r}\right. & \left.\frac{\partial}{\partial \theta}\right)
\end{array}\right) \operatorname{Im} \phi_{1 k} \quad k=1,2, \\
& -0=2 m r_{0}^{2} \tau_{0} \cos 30, \quad \mathrm{fc} \quad=0
\end{aligned}
$$


Second approximation.

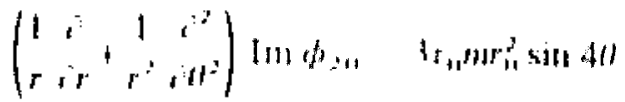

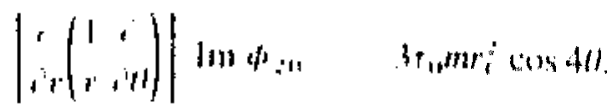

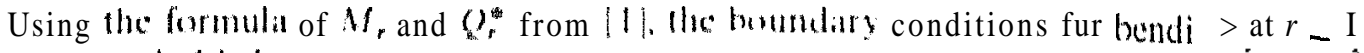
are recorded beluw

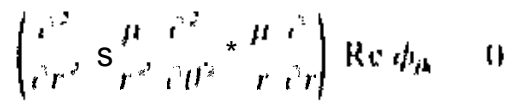

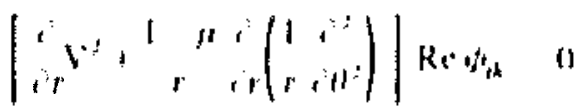

$10,1.1$

h $\quad 0,1,2,1,4$.

\section{ZEROIH AIPROXIMATION}

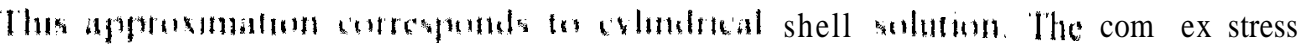
func(t) forf the is

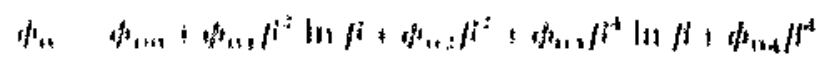

where

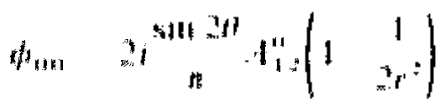

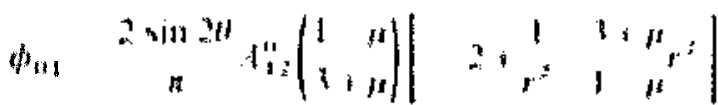

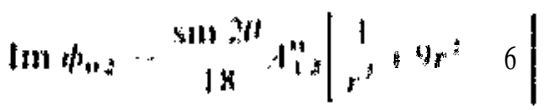

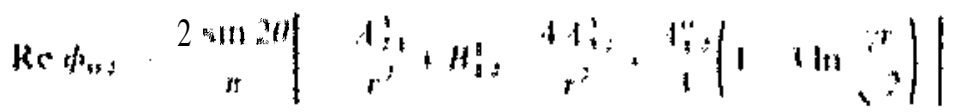

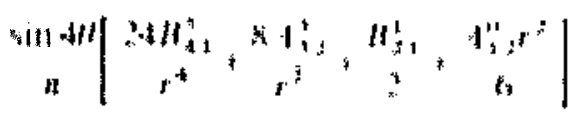

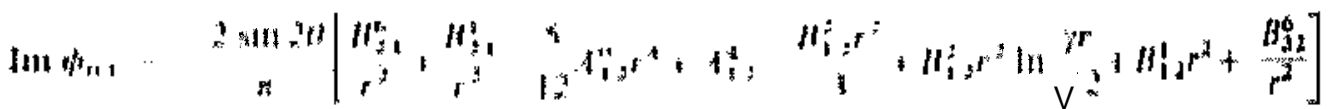

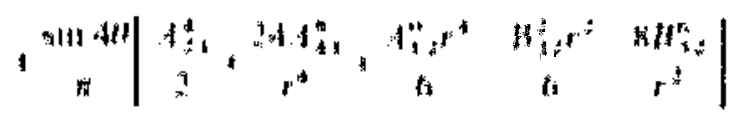

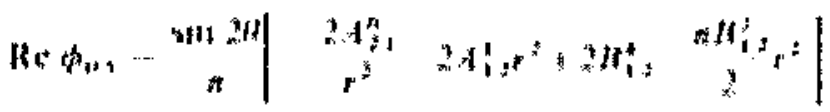




$$
\begin{aligned}
& \operatorname{Im} \phi_{04}=\frac{\sin 20}{\pi}\left[-\frac{2 B_{21}^{5}}{r^{2}}-r^{2} B_{21}^{1}\left(\frac{1}{48}+\frac{1}{2} \ln \frac{y r}{\sqrt{2}}\right)+\frac{6 B_{A}^{5}}{2}+\frac{\pi}{2} A_{12}^{1} r^{2}-2 A_{13}^{3}+\frac{2}{3} B_{12}^{1}\right. \\
& \left.\times\left(1-3 \ln \frac{\gamma r}{\sqrt{2}}\right)-\frac{8 B_{32}^{5}}{r^{2}}+\frac{A_{12}^{0} r^{4}}{240}\left(-185+200 \ln \frac{\gamma r}{\sqrt{ } 2}\right)\right]+\frac{\sin 4 \theta}{\pi}\left[\frac{A_{21}^{3}}{2}-\frac{B_{21}^{1} r^{2}}{6}\right. \\
& \left.+\frac{24 A_{41}^{7}}{r^{4}}+\frac{8 R_{41}^{5}}{r^{2}} \cdot \frac{A_{\mathrm{I} 2}^{6} r^{4}}{240}\left(16+40 \ln \frac{\gamma r}{\sqrt{2}}\right)-\frac{B_{12}^{1} r^{2}}{6}-\frac{8 B_{32}^{5}}{r^{2}}+\frac{192 A_{52}^{9}}{r^{7}}\right]_{\rfloor}-\frac{\sin 60}{\pi} \\
& \times\left[\frac{B_{21}^{1} r^{2}}{48}+\frac{6 B_{41}^{5}}{r^{2}}{ }_{\mathrm{r}} \frac{A_{12}^{0} r^{4}}{240}+\frac{2}{3} A^{2} \frac{1}{3} 2+\frac{192}{r^{4}} A_{52}^{y}-\frac{960}{r^{6}} A_{61}^{4}\right]
\end{aligned}
$$$$
\operatorname{Re} \phi_{04}=\frac{\sin 2 \theta}{\pi}\left[-\frac{2 A_{21}^{5}}{r^{2}}-\frac{\pi B_{21}^{1}}{8} r^{2}+\frac{5 \pi}{24} A_{12}^{0} r^{4}+\frac{2}{3} A_{12}^{1} r^{2}\right.
$$$$
\left.-2 A_{12}^{1} r^{2} \ln \frac{\gamma r}{\sqrt{2}}-\frac{\pi B_{12}^{1}}{2} r^{2}+2 B_{12}^{3}-\frac{8 A_{32}^{5}}{r^{2}}\right]
$$$$
+\sin 40\left[\frac{B_{2}^{3} \cdot}{2}-\frac{24 B_{41}^{7}}{r^{4}}+\frac{\pi A_{12}^{0} r^{4}}{24}-\frac{A_{12}^{1} r^{2}}{6}-\frac{8 A_{32}^{5}}{r^{2}}\right]
$$

where, the different $A^{s}$ and $B^{s}$ with subscript and superscript are given below:

$$
\begin{aligned}
& A_{12}^{0}=\frac{m \pi}{2} r_{0}^{2} \tau_{0}, \quad A_{12}^{1}=\frac{\pi}{6} A_{12}^{0}, \quad A_{21}^{4}=\frac{\mu-1}{\mu+3} A_{12}^{0}
\end{aligned}
$$

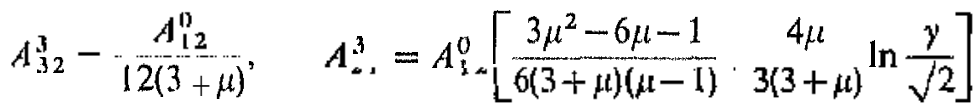

$$
\begin{aligned}
& A_{41}^{7}=\frac{A_{12}^{0}}{480}\left[\frac{10(9 \mu-1)}{3(3+\mu)} \ln \frac{\gamma}{\sqrt{2}}+\frac{4034 \mu^{2}+11,860 \mu-18,3307}{360(3+\mu)(\mu-1) \quad \mathrm{J}}\right. \\
& A_{12}^{3}=\frac{A_{12}^{0}}{8}\left[-\frac{2 \pi^{2}}{3}+\frac{18(5-\mu)}{3+\mu}\left(\ln \frac{\gamma}{\sqrt{2}}\right)^{2}-\frac{58 \mu+304}{3(3+\mu)} \ln \frac{\gamma}{\sqrt{2}}-\frac{948 \mu^{2}-2141 \mu+17,809}{360(3+\mu)(\mu-1)}\right] \\
& A_{52}^{4}=\frac{1-\mu}{3+\mu} \frac{A_{12}^{0}}{24 \times 384}, \quad A_{6 \mathrm{~L}}^{4}=-\frac{A_{12}^{0}}{42 \times 960} \frac{63 \mu+49}{240(\mu+3)} \\
& B_{21}^{3}=-\frac{\pi}{36} A_{12}^{0}, \quad B_{21}^{1}=-\frac{A_{12}^{0}}{2}, \quad B_{12}^{2}=-\frac{2(1-\mu)}{3+\mu} A_{12}^{0} \\
& B_{12}^{1}=A_{12}^{0}\left[\frac{\mu-15}{6(\mu+3)}+\frac{5-\mu}{3+\mu} \ln \frac{\gamma^{1}}{\sqrt{2}}\right], \quad B_{41}^{5}=\frac{A_{12}^{0}}{288} \frac{\mu-3}{\mu+3} \\
& B_{32}^{5}=\frac{A_{12}^{0}}{48}\left[\frac{79 \mu^{2}+188 \mu-327}{15(3+\mu)(\mu-1)}+\frac{3(5 \mu-1)}{3+\mu} \ln \frac{\gamma}{\sqrt{2}}\right] \\
& B_{21}^{5}=\frac{A_{12}^{0}}{12}\left[\left(\ln \frac{\gamma}{\sqrt{2}}\right)^{2} \frac{10(5-\mu)}{3+\mu}-\ln \frac{\gamma}{\sqrt{2}} \frac{59 \mu+357}{6(3+\mu)}-\frac{\pi^{2}}{2}+\frac{97 \mu+279}{16(3+\mu)}\right] .
\end{aligned}
$$




\subsection{Mimbrame sfresues}

The membrane stress $a_{m 1}^{\prime}$ is expressed as

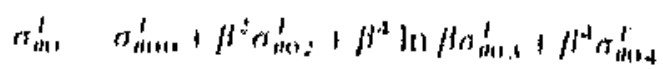

where $\sigma_{n i n}$, arc dectermined from the formula

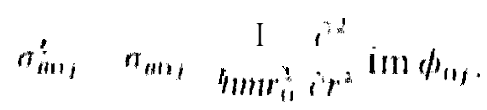

The general expression lor $\|_{b u}^{t}$ is not given luere as it is lengthy one. Ho expression at ;• I is recorded as belisw

ever, the

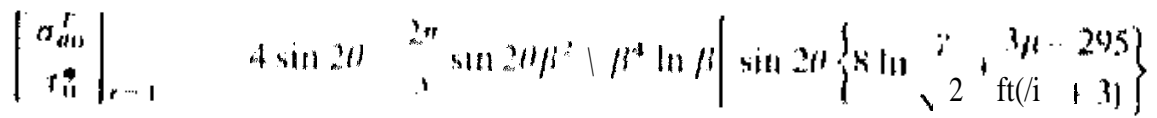

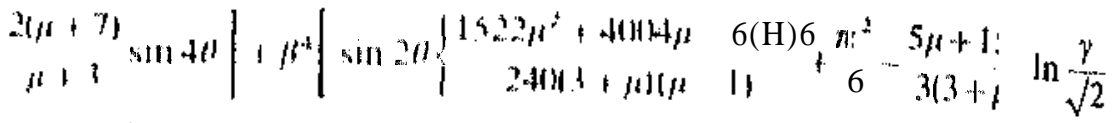

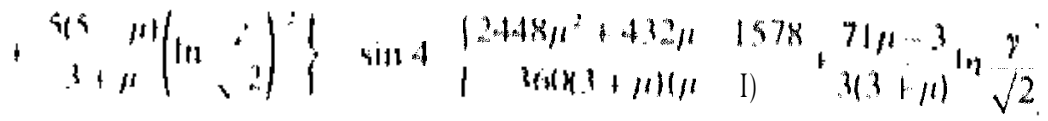

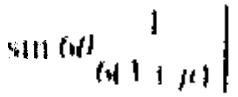

where $t_{*}^{*} \quad r_{11} h$

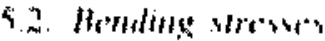

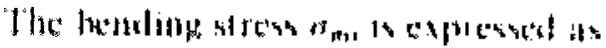

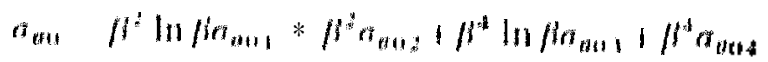

where $\mathrm{ff}_{\mathrm{m}}$ / ate ablaticel from the formula

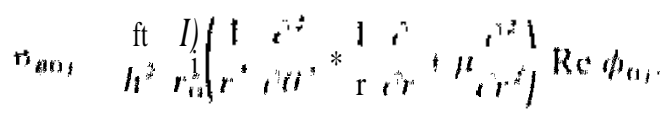

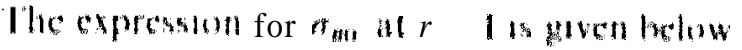

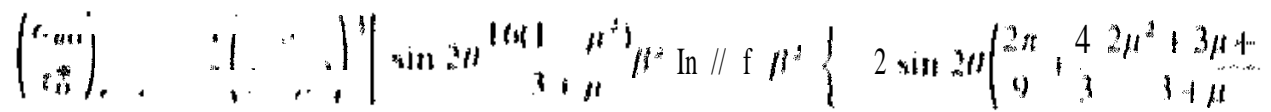

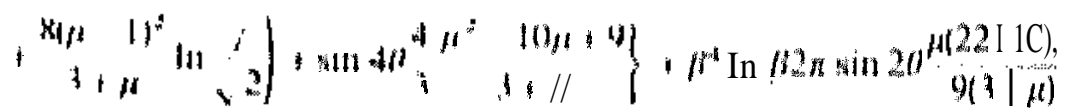

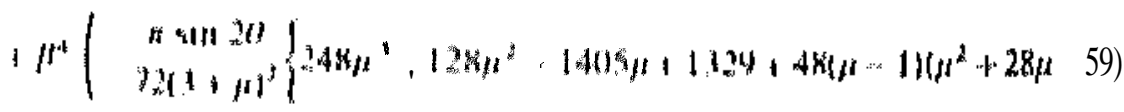

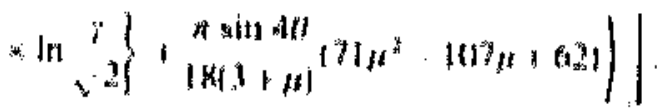




\section{FIRST APPROXIMATION}

The governing equation for the approximation is

$$
\nabla^{4} \phi_{1}+8 i \beta^{2} \frac{\partial^{2} \phi_{1}}{\partial x^{2}}=\beta^{2} i L_{1} \phi_{0}
$$

Replacing $\phi_{0}$ in the above equation by its expansion, we obtain

$$
\nabla^{4} \phi_{1}+8 i \beta^{2} \frac{\partial^{2} \phi_{2}}{\partial x^{2}}=i L_{1}\left[\beta^{2} \phi_{00}+\alpha\left(\beta^{4} \ln \beta\right)\right] .
$$

Since we are neglecting the terms of order of $\beta^{4} \ln \beta$ in the expansion of $\phi_{1}$, the particular solution of the above equation can also be terminated at $\beta^{2}$ order terms.

The solution of the above equation, $\phi_{1}$ can be expressed as

$$
\phi_{1}=\phi_{1}^{P}+\phi_{1}^{c}
$$

where $\phi_{1}^{l}$ is particular solution, and

$\phi_{1}^{c}$ is complementary solution.

If can be assumed as $\phi_{1}^{p}-\phi_{12}^{p} \beta^{2}+0\left(\beta^{4} \ln (\beta)\right.$.

Substituting the assumed expansion of $\phi_{1}^{p}$ in the governing equation and equating the coefficients of $\beta^{2}$, we have

$$
\nabla^{4} \phi_{12}^{p}=i L_{1} \phi_{00}
$$

The above equation is rewritten after substituting $\phi_{00}$ from 0th approximation and operating it by the differential operator $L_{1}$ as defined earlier.

$$
\nabla^{4} \phi_{12}^{r}=-48 B_{21}^{\prime}\left[-3 r^{-3} \sin 5 \theta+r^{-3} \sin 3 \theta\right]+\frac{16 A_{12}^{0}}{\pi r}(3 \sin 5 \theta-2 \sin 3 \theta-\sin 6) .
$$

Solving the above equation,

$$
\phi_{12}^{\prime \prime}=\frac{B_{21}^{i}}{4 \pi} r(\sin 50-3 \sin 30)+-{ }_{\pi}^{n}\left[\frac{r^{3}}{8} \sin 50+{ }_{3}^{2} r^{3} \ln r \sin 3 \theta-\frac{r^{3} \sin \theta}{4}\left(4 \ln r^{\prime}-3\right)\right]
$$

Complementarysolution. The complementary solution $\phi_{\mathrm{i}} \ddot{\text { is assumed as }}$

$$
\phi_{1}^{c}=\phi_{10}^{c}+\beta^{2} \ln \beta \phi_{11}^{c}+\beta^{2} \phi_{12}^{c}
$$

combining the assumed complementary solution and particular solutions, $\phi_{1}$ is determined by using the appropriate boundary conditions.

$$
\phi_{1}=\phi_{10}+\beta^{2} \ln \beta \phi_{12}+\beta^{2} \phi_{12}
$$

where

$$
\begin{aligned}
\phi_{10} & =\frac{2 \sin 3 \theta}{\pi} A_{11}^{2}\left(\frac{1}{r}-\frac{2}{3 r^{3}}\right) \\
\phi_{11} & =0 \\
\operatorname{Im} \phi_{12} & =\frac{A_{1}^{2} \cdot r}{2} \sin \theta
\end{aligned}
$$




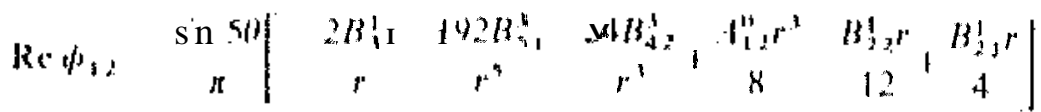

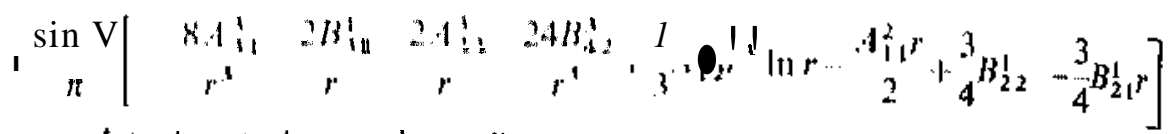

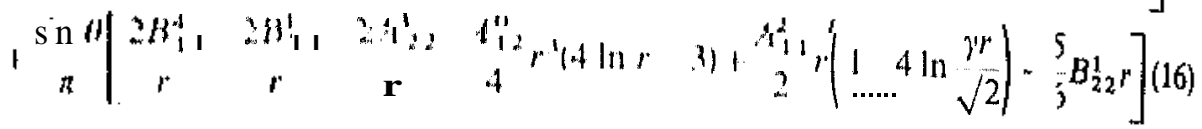

where

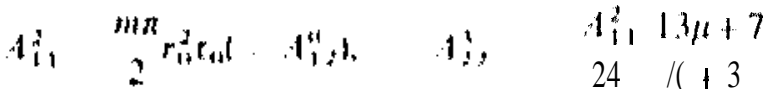

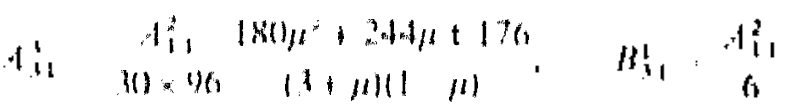

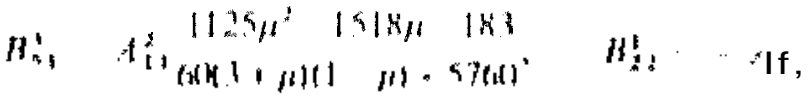

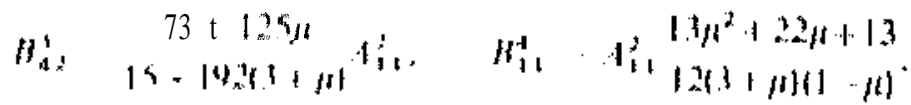

\section{C>, I. Mrmbrallewrisu's}

flic membrane stress al mexpencel is

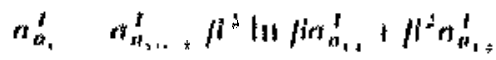

where $a_{i, j}$ is uhlatucd from

where $t: 0$, I. 2

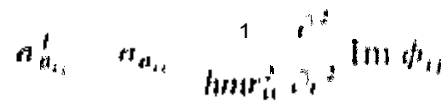

The general expression of $\boldsymbol{n}_{\boldsymbol{n}_{1}}^{\prime}$ in

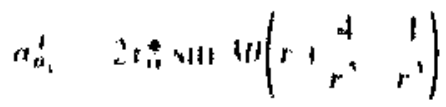

Substifutuger - I m the above cquatuan, we whan

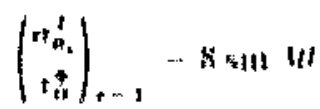

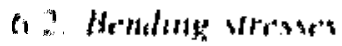

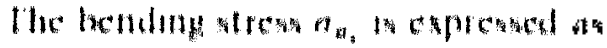

$$
n_{\mathbf{w}_{1}}--\|^{2} n_{\mathbf{n}_{1},}
$$

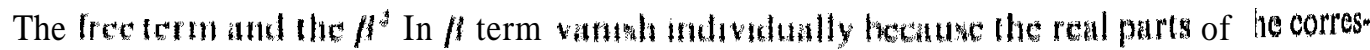

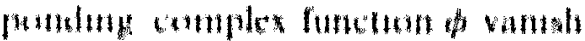


The (70, at $r=1$ is recorded below:

$$
\begin{aligned}
\left(\frac{\sigma_{v_{1}}}{\tau_{n}^{*}}\right)_{r=1}= & \beta^{2}\left(\frac{3}{1-\mu^{2}}\right)^{\frac{1}{2}}\left[\sin 50 \frac{115 \mu^{2}-144 \mu-259}{15(3+\mu)}+\sin 3 \theta \frac{-\mu^{2}+60 \mu+61}{3(3+\mu)}\right. \\
& -(4 \mu+5) \sin 0 \frac{7}{]}
\end{aligned}
$$

\section{SECOND APPROXIMATION}

The governing equation of $\phi_{2}$ is

$$
\nabla^{4} \phi_{2}+8 i \beta^{2} \frac{\partial^{2} \phi_{2}}{\partial x^{2}}=i \beta^{2}\left(L_{1} \phi+L_{2} \phi_{2}\right)
$$

The particular solution of the above equation will be starting from the terra containing $f t^{2}$, since $f t^{2}$ order terms are not to be considered for this approximation, there is no need of determining the particular solution. The complementary solution also will be confined to free term i.e. $\phi_{20}{ }^{\circ}$

$\phi_{2}$ is recorded as below:

$$
\phi_{2}=i-\frac{m m^{2}}{4} \tau_{0} \sin 4 v\left(\frac{2}{r^{4}}-\frac{4}{r^{2}}\right) .
$$

The membrane stress $\sigma_{n_{2}}^{r}$ is now computed from

$$
\sigma_{\delta_{2}}^{T^{r}}=\overline{\sigma_{0_{2}}}-\frac{1}{h m r_{0}^{2}} \frac{\partial^{2}}{\partial r^{2}} \operatorname{Im} \phi_{2}
$$

and recorded as

$$
\sigma_{\theta_{2}}^{\tau_{2}}=-\tau_{0}^{*} \sin 40\left(\frac{15}{r^{6}}-\frac{6}{r^{4}}+3 r^{2}\right)
$$

Substituting $r=1$ in the above expression,

$$
\frac{\sigma_{0_{2}}^{T}}{\tau_{0}^{*}}=-12 \sin 40
$$

Bending stress is vanishing as $\operatorname{Re} \phi_{2}=0$.

\section{COMPLETE SOLUTION}

The complete solution to the problem up to the terms containing $\varepsilon^{2}$ is now written by adding Oth, 1st and 2nd approximation solution. The total stress $\sigma_{\theta}^{T}$ is expressed as the sum of total membrane and bending stresses namely

$$
\sigma_{0}^{T}-\sigma_{0(m)}^{T}+\sigma_{0 b}^{T}
$$

where $m$ and $b$ denote membrane and bending solutions. Now $f f i_{m}$ is obtained from the following

$$
\sigma_{\theta_{m 1}}^{T}=\sigma_{\theta_{m+Q}}^{T}+8 \sigma_{\theta_{m+1}}^{T}+\varepsilon^{2} \sigma_{\theta_{m 2}}^{T} .
$$




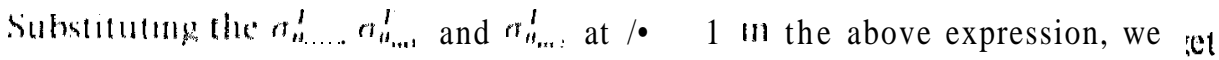

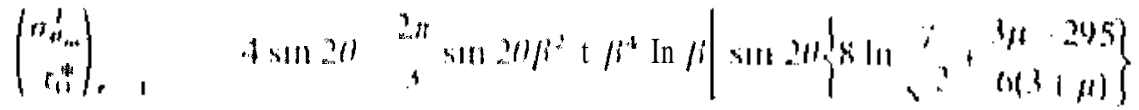

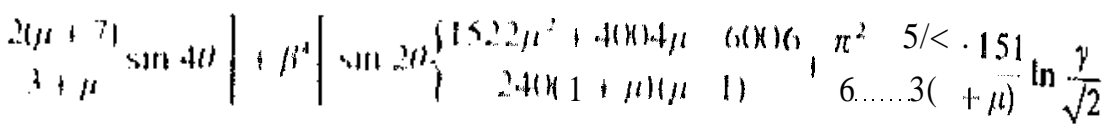

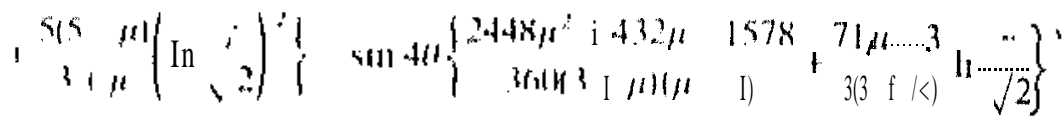

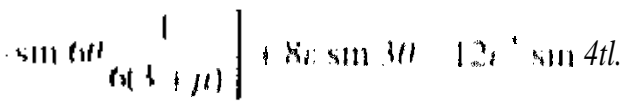

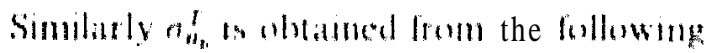

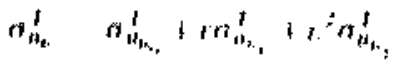

As

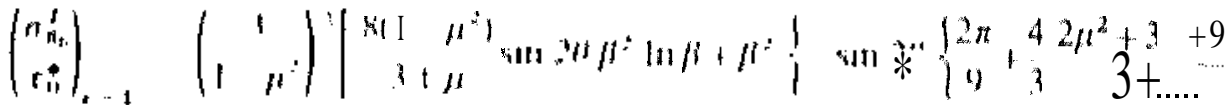

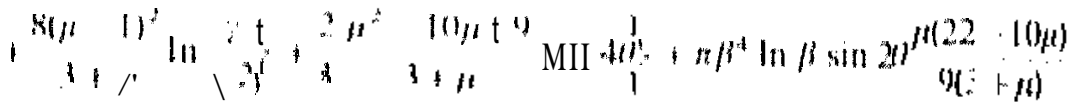

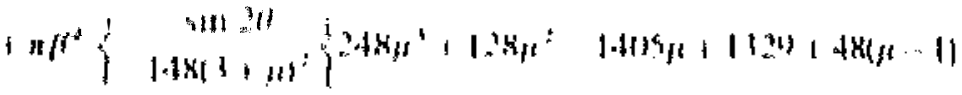

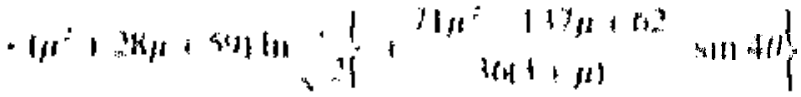

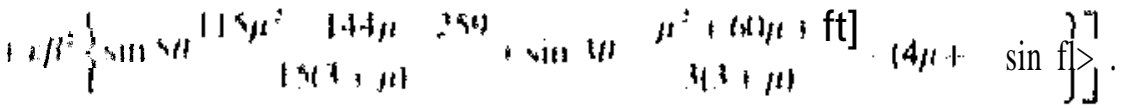

\section{9. $\operatorname{DIN}(\mathrm{isis} I 0) \mathrm{N}$}

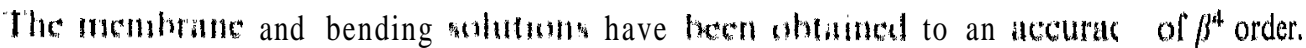

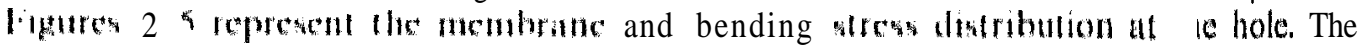

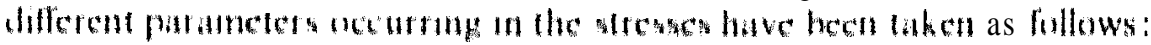

$$
\text { R.....J(k) }
$$

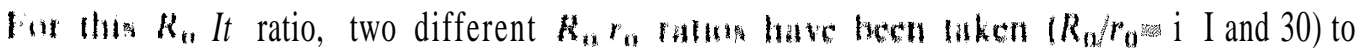

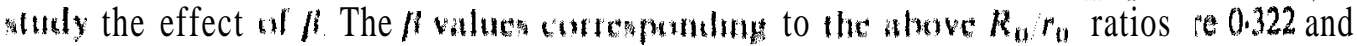

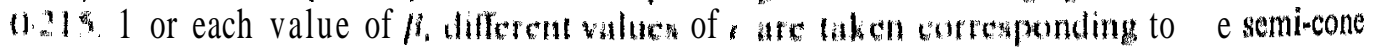

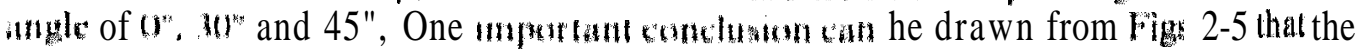

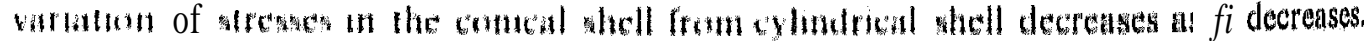

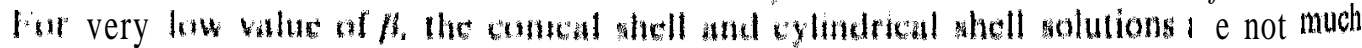

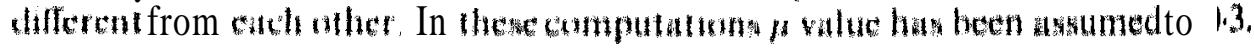


Stresses around a circular hole in a shallow conical shell with torsional loading

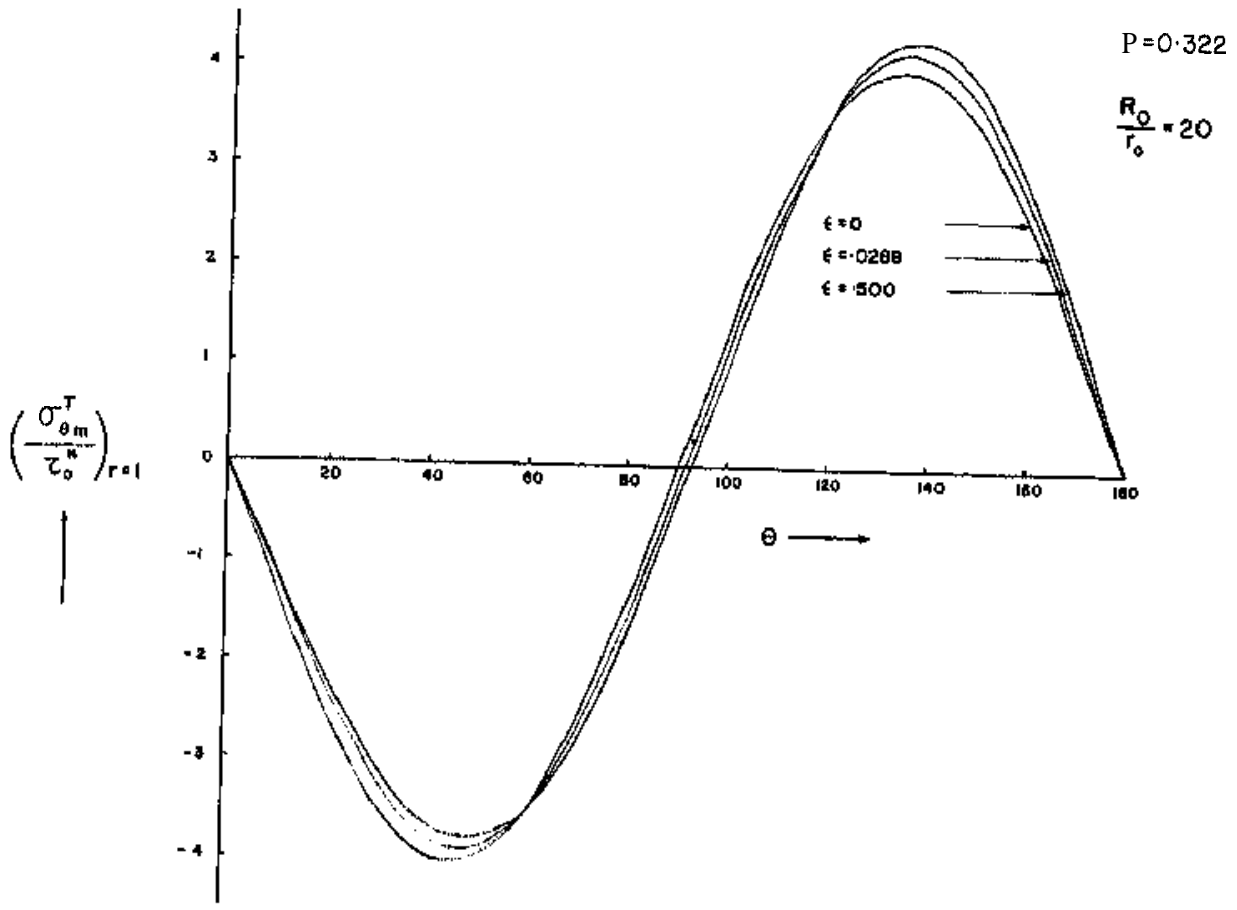

Fici. 2. Membrane stresses due to torsional load,

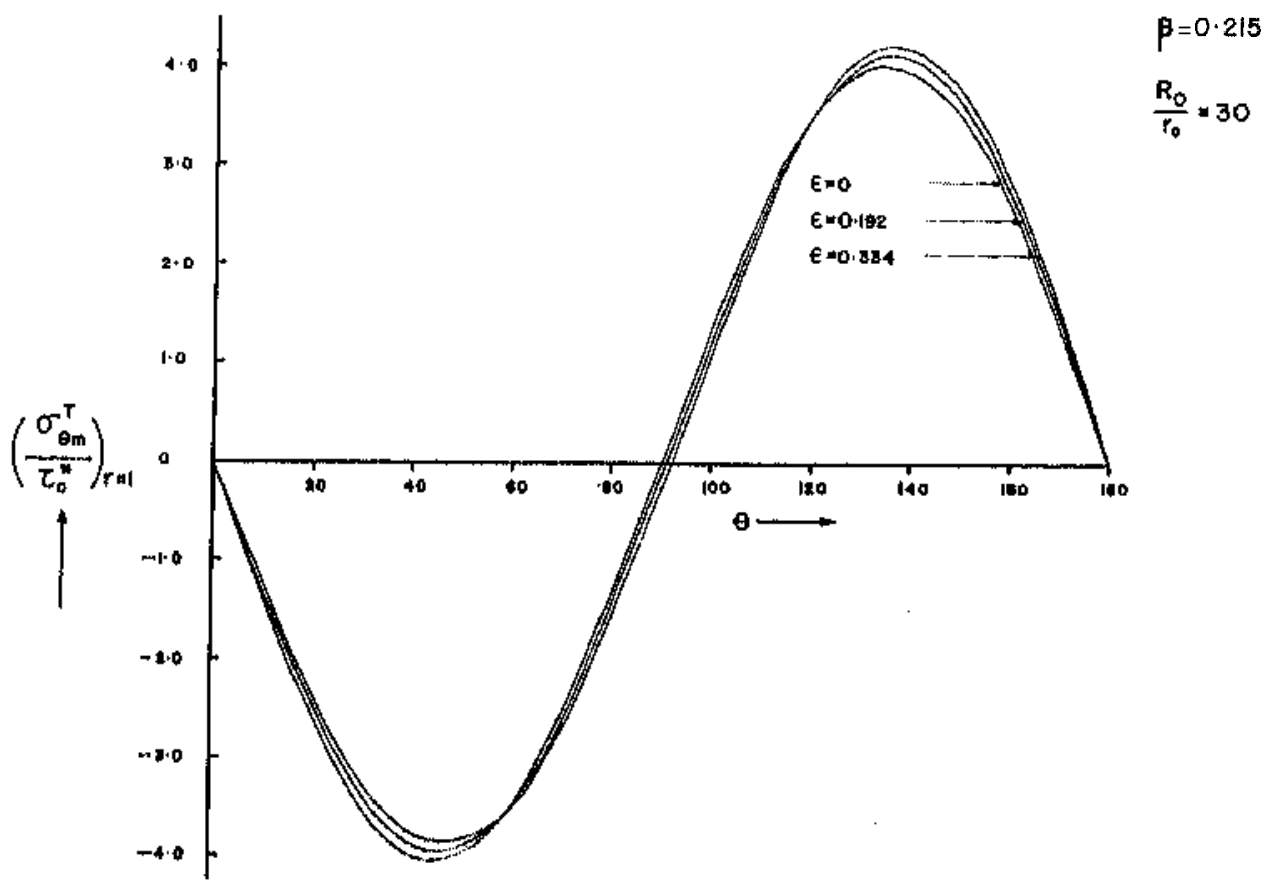

Fia. 3, Membrane stresses due to torsional load. 


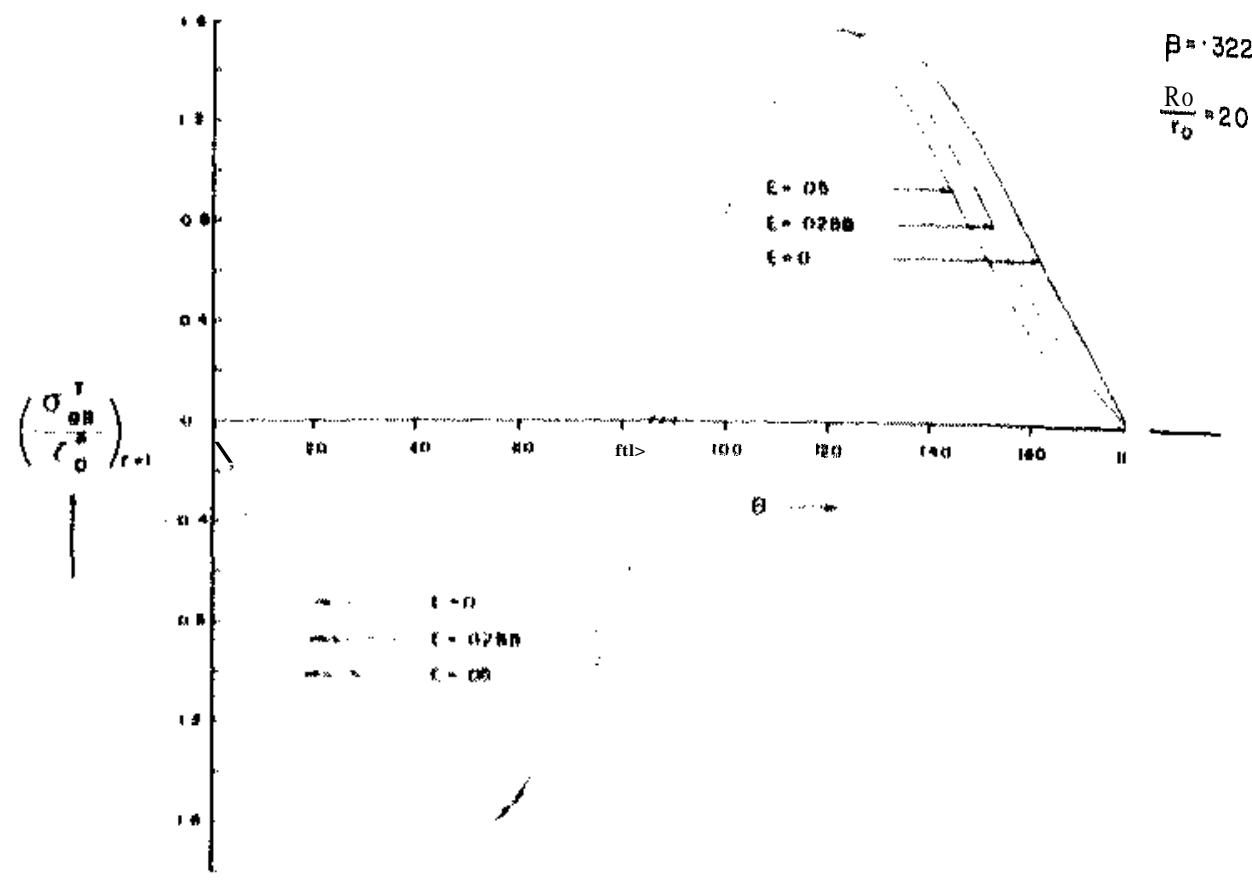

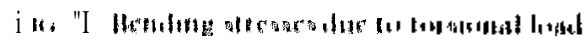

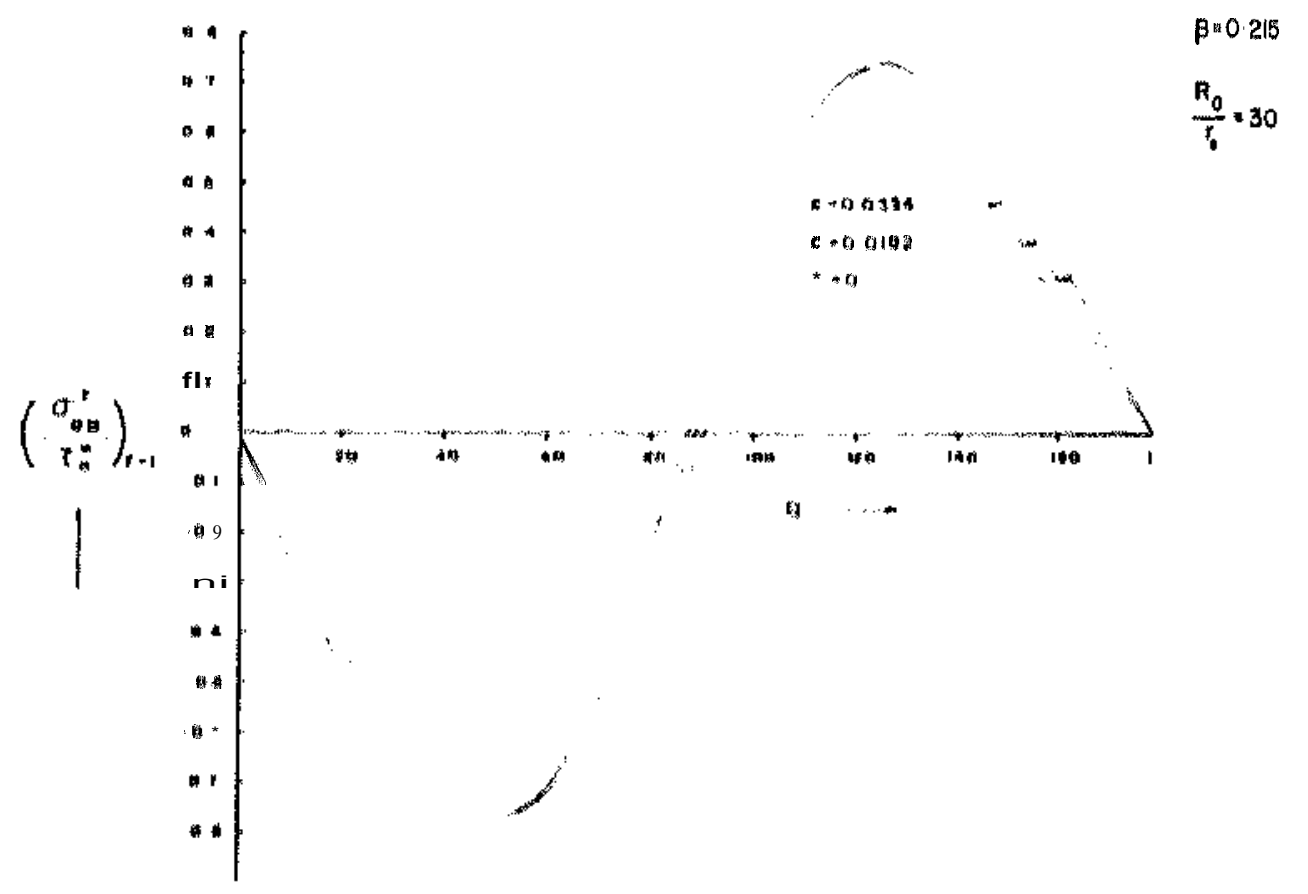

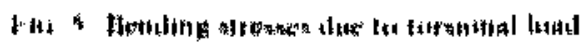


Ackmowledgeme'nt Thanks are due to Mr. M. V. V. Murthy for his valuable suggestions.

\section{REFERENCES}

[1] B. BASAVA Raju, M. V. I MURThHY and Ramesh Cram, Tresses around a circular hole in a shallow
conical shell. Imt. J. Solids Struct. 4,993 1011 (1968)

[2] A. I. LuRIE, Statics of Thin-WalledElastic Shells State FrübitishingFfrotsse \&f Technical and Theoretical

[3] A. N. Guz, (Kiev), Stress in a conical shell weakened by a circular hole. NAL Technical Translation, TT-1.

\section{APPENDIX}

Derivation of basic stresses $\bar{N}_{r}, N_{0}$ and $\bar{N}_{r 0}$

Considering the section of a conical shell in the second principal direction subjected to torsion it $m$, $h$ shown $h$ equa $n$ of equilibrium that the membrane stresses are
given by

$$
\begin{aligned}
\bar{N}_{s \psi} & =\tau_{0} /\left(1+2 \varepsilon r \cos 0+\varepsilon^{2} r^{2}\right) \\
\bar{N}_{s} & =0, \bar{N}_{\psi}=\mathrm{O}
\end{aligned}
$$

where $\tau_{0}=T^{\prime} /\left(2 \pi R_{0}^{2}\right), T$ is applied torsional load

Now $\bar{N}_{r}, \bar{N}_{0}$ and $\bar{N}_{r \theta}$ will be computed from the above stresses by using the transformations

$$
\begin{aligned}
\bar{N}_{r} & =\bar{N}_{s \psi} \sin 2 \lambda \\
\bar{N}_{0} & =-\bar{N}_{s \psi} \sin 2 \lambda \\
f l_{r l l} & =\bar{N}_{s \psi} \cos 2 \lambda
\end{aligned}
$$

where $\lambda$ is the angle between $s$ and $\mathrm{r}$ direction [Fig. 1(a)]. It can be shown that

$$
\begin{aligned}
\sin \angle \lambda & =\frac{\sin 2 \theta+2 \varepsilon r \sin \theta}{1+2 \varepsilon r \cos \theta+\varepsilon^{2} r^{2}} \\
\cos 2 \lambda & =\frac{\cos 2 \theta+2 \varepsilon r \cos \theta+\varepsilon r^{2}}{1+2 \varepsilon r \cos \theta+\varepsilon^{2} r^{2}} .
\end{aligned}
$$

By substituting (A-3) and (A-1) in (A-2), we obtain

$$
\begin{gathered}
N_{r}=\tau_{0}(\sin 20-2 \varepsilon r \sin 39+3 r \mathrm{~V} \sin 40) \\
\bar{N}_{\theta}=-\tau_{0}(\sin 20 \sim 2 e r \sin 30+3 r \mathrm{~V} \sin 40) \\
f f l_{r n}=\tau_{0}\left(\cos 20-2 \varepsilon r \cos 30+3 r^{2} \varepsilon^{2} \cos 40\right)
\end{gathered}
$$

(Received 26 August 1969)

Абетрант Даноте аналитнческе решения для напряжений, В конической оболочке с круглым

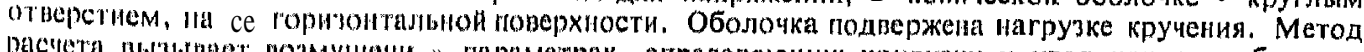

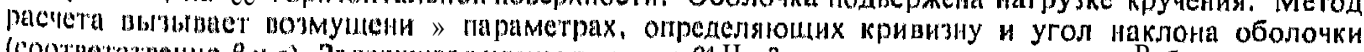

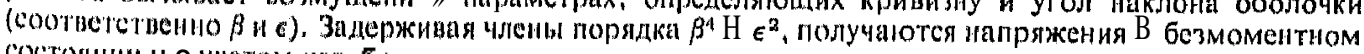
состоянин н с учетом изтиба. 